\title{
Crystal structure of cyprodinil
}

\author{
Youngeun Jeon, Gihaeng Kang, Seonghwa Cho and
}

Tae Ho Kim*

Department of Chemistry and Research Institute of Natural Sciences, Gyeongsang National University, Jinju 660-701, Republic of Korea. *Correspondence e-mail:

thkim@gnu.ac.kr

Received 24 November 2014; accepted 25 November 2014

Edited by P. C. Healy, Griffith University, Australia

In the title compound, $\mathrm{C}_{14} \mathrm{H}_{15} \mathrm{~N}_{3}$ (systematic name: 4-cyclopropyl-6-methyl- $N$-phenylpyrimidin-2-amine), which is the anilinopyrimidine fungicide cyprodinil, the dihedral angles between the planes of the central pyrimidine ring and the terminal phenyl ring and the mean plane of the cyclopropane ring system are $14.52(11)$ and $88.79(10)^{\circ}$, respectively. In the crystal, weak $\pi-\pi$ interactions [3.8551 (11) $\AA$ ] connect the dimers into chains along the $b$-axis direction.

Keywords: crystal structure; cyprodinil; pyrimidin-2-amine; fungicide; hydrogen bonding; $\pi-\pi$ interactions.

CCDC reference: 1035821

\section{Related literature}

For information on the fungicidal properties of the title compound, see: Sapp et al. (2003). For a related crystal structure, see: Kang et al. (2014).

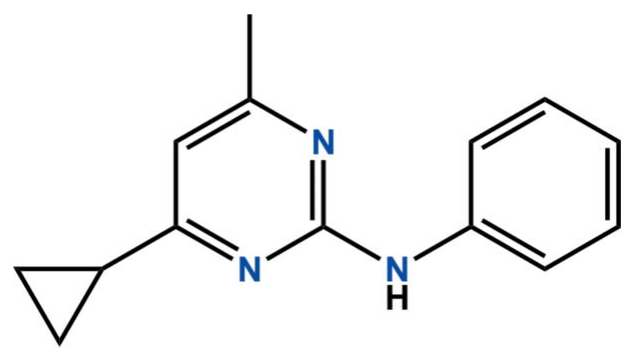

\subsection{Refinement \\ $R\left[F^{2}>2 \sigma\left(F^{2}\right)\right]=0.056$ \\ $w R\left(F^{2}\right)=0.166$ \\ $S=1.14$ \\ 2864 reflections}

$$
\begin{aligned}
& V=1163.99(8) \AA^{3} \\
& Z=4 \\
& \text { Mo } K \alpha \text { radiation } \\
& \mu=0.08 \mathrm{~mm}^{-1} \\
& T=173 \mathrm{~K} \\
& 0.45 \times 0.22 \times 0.18 \mathrm{~mm}
\end{aligned}
$$

18116 measured reflections 2864 independent reflections 2463 reflections with $I>2 \sigma(I)$ $R_{\text {int }}=0.056$

Data collection: APEX2 (Bruker, 2009); cell refinement: SAINT (Bruker, 2009); data reduction: $S A I N T$; program(s) used to solve structure: SHELXTL (Sheldrick, 2008); program(s) used to refine structure: SHELXTL; molecular graphics: DIAMOND (Brandenburg, 2010); software used to prepare material for publication: SHELXTL.

\section{Acknowledgements}

This research was supported by the Basic Science Research Program through the National Research Foundation of Korea (NRF) funded by the Ministry of Education, Science and Technology (No. 2012R1A1B3003337).

Supporting information for this paper is available from the IUCr electronic archives (Reference: HG5422).

\section{References}

Brandenburg, K. (2010). DIAMOND. Crystal Impact GbR, Bonn, Germany. Bruker (2009). APEX2, SAINT and SADABS. Bruker AXS Inc., Madison, Wisconsin, USA.

Kang, G., Jeon, Y., Lee, S. \& Kim, T. H. (2014). Acta Cryst. E70, o1265.

Sapp, M., Ertunc, T., Bringmann, I., Schaffer, A. \& Schmidt, S. (2003). Pest Manag. Sci. 60, 65-74.

Sheldrick, G. M. (2008). Acta Cryst. A64, 112-122. 


\section{supporting information}

Acta Cryst. (2015). E71, o5 [https://doi.org/10.1107/S2056989014025742]

\section{Crystal structure of cyprodinil}

\section{Youngeun Jeon, Gihaeng Kang, Seonghwa Cho and Tae Ho Kim}

\section{S1. Comment}

Cyprodinil, $\mathrm{C}_{14} \mathrm{H}_{15} \mathrm{~N}_{3}$, is a systemic pyrimidine fungicide for foliar applications on cereals and strawberries against plant pathogenic fungi (Sapp et al., 2003). Its crystal structure is reported herein. In this compound (Scheme 1, Fig. 1), the dihedral angles between the central pyrimidine ring and the terminal phenyl ring and mean plane of cyclopropane ring system are $14.52(11)$ and $88.79(10)^{\circ}$. All bond lengths and bond angles are normal and comparable to those observed in the crystal structure of a similar compound (Kang et al., 2014).

In the crystal structure (Fig. 2), The crystal structure is stabilized by weak intermolecular $\pi-\pi$ interaction between the pyrimidine ring and terminal phenyl ring systems $\left[C \mathrm{~g} 1 \cdots C \mathrm{~g} 22^{\mathrm{ii}}, 3.8552(11) \AA\right]$ are present $(C \mathrm{~g} 1$ and $C \mathrm{~g} 2$ are the centroids of the $\mathrm{C} 1-\mathrm{C} 6$ and $\mathrm{C} 7-\mathrm{N} 2-\mathrm{C} 8-\mathrm{C} 9-\mathrm{C} 10-\mathrm{N} 3$ rings, respectively) [for symmetry codes: (ii), $x, y-1, z]$.

\section{S2. Experimental}

The title compound was purchased from the Chem Servies Company. Slow evaporation of a solution in $\mathrm{CH}_{2} \mathrm{Cl}_{2}$ gave single crystals suitable for X-ray analysis.

\section{S3. Refinement}

All H-atoms were positioned geometrically and refined using a riding model with $\mathrm{d}(\mathrm{N}-\mathrm{H})=0.88 \AA, U_{\text {iso }}=1.2 U_{\text {eq }}(\mathrm{C})$ for amine group, $\mathrm{d}(\mathrm{C}-\mathrm{H})=0.98 \AA, U_{\text {iso }}=1.5 U_{\text {eq }}(\mathrm{C})$ for methyl group, $\mathrm{d}(\mathrm{C}-\mathrm{H})=0.99 \AA, U_{\text {iso }}=1.2 U_{\text {eq }}(\mathrm{C})$ for $\mathrm{Csp} p^{3}-\mathrm{H}, \mathrm{d}(\mathrm{C}$ $-\mathrm{H})=1.00 \AA, U_{\text {iso }}=1.2 U_{\mathrm{eq}}(\mathrm{C})$ for $\mathrm{Csp} p^{3}-\mathrm{H}$, and $\mathrm{d}(\mathrm{C}-\mathrm{H})=0.95 \AA, U_{\text {iso }}=1.2 U_{\mathrm{eq}}(\mathrm{C})$ for aromatic $\mathrm{C}-\mathrm{H}$. 


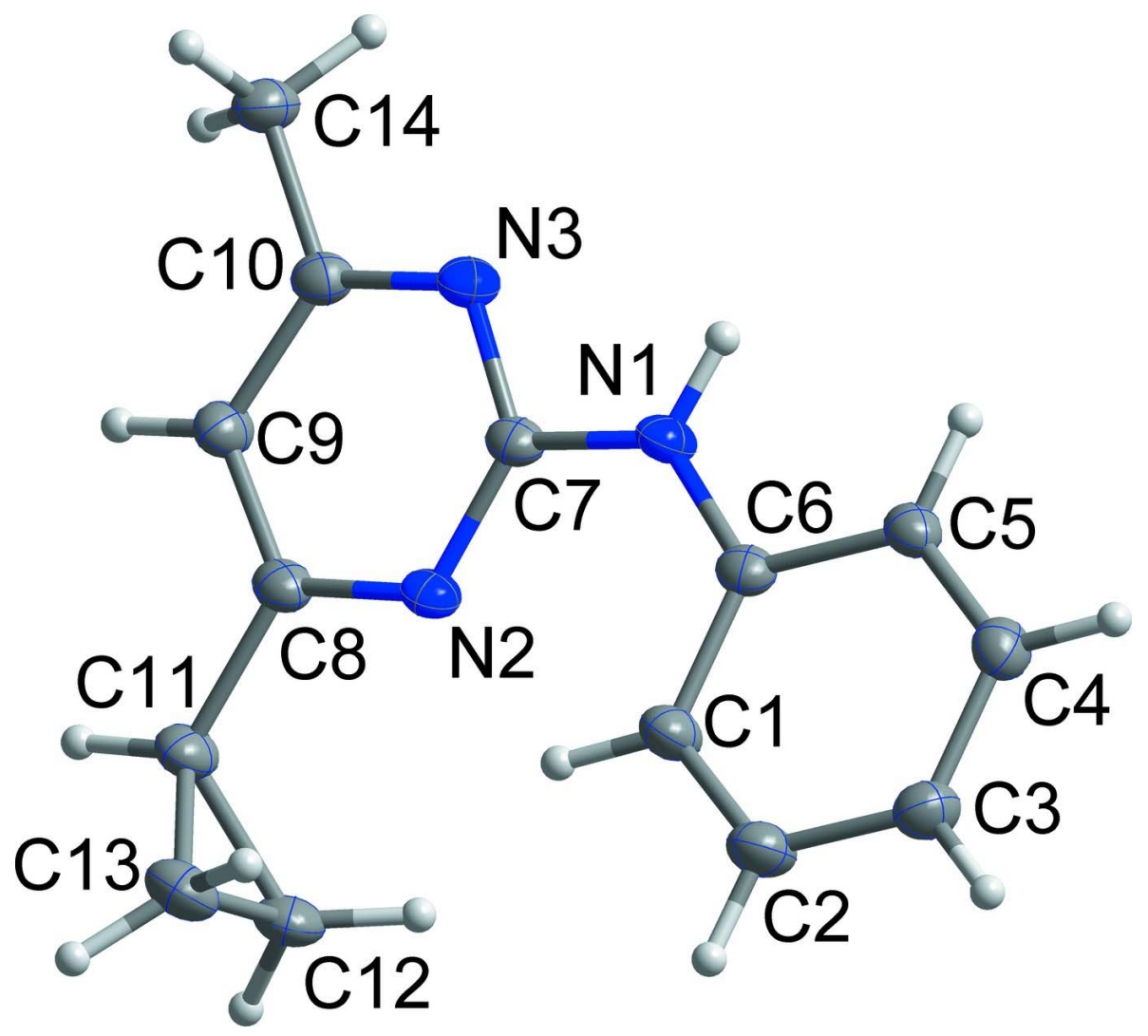

Figure 1

The asymmetric unit of the title compound with the atom numbering scheme. Displacement ellipsoids are drawn at the $50 \%$ probability level. $\mathrm{H}$ atoms are shown as small spheres of arbitrary radius. 


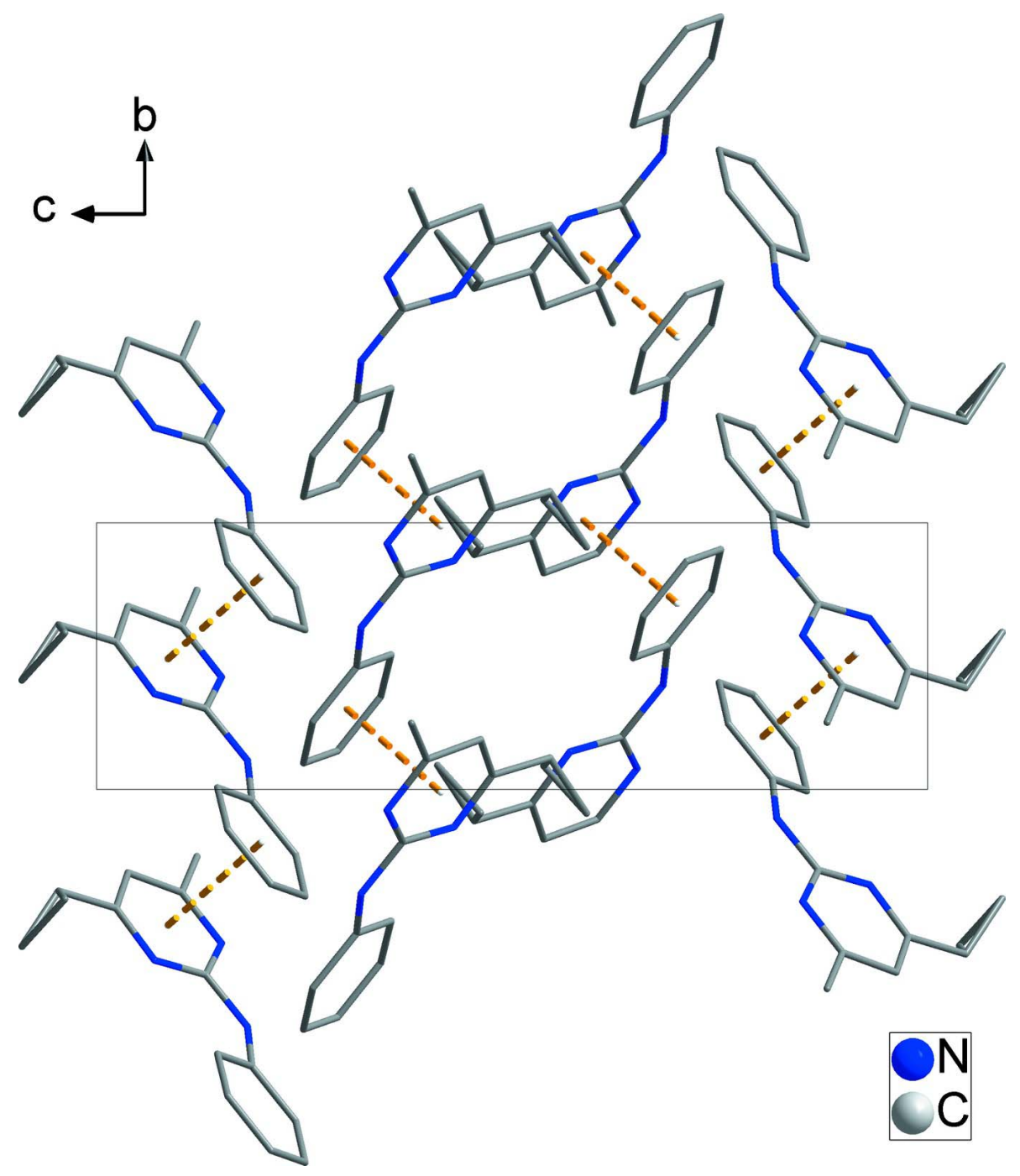

Figure 2

Crystal packing viewed along the $a$ axis. The weak $\pi-\pi$ interactions are shown as dashed lines.

4-Cyclopropyl-6-methyl- $N$-phenylpyrimidin-2-amine

Crystal data

$\mathrm{C}_{14} \mathrm{H}_{15} \mathrm{~N}_{3}$

$M_{r}=225.29$

Monoclinic, $P 2_{1} / c$

Hall symbol: -P 2ybc

$a=13.1920$ (6) $\AA$

$b=5.3176(2) \AA$

$c=16.8641$ (7) $\AA$

$\beta=100.288(2)^{\circ}$

$V=1163.99(8) \AA^{3}$

$Z=4$
$F(000)=480$

$D_{\mathrm{x}}=1.286 \mathrm{Mg} \mathrm{m}^{-3}$

Mo $K \alpha$ radiation, $\lambda=0.71073 \AA$

Cell parameters from 8168 reflections

$\theta=2.5-28.2^{\circ}$

$\mu=0.08 \mathrm{~mm}^{-1}$

$T=173 \mathrm{~K}$

Block, colourless

$0.45 \times 0.22 \times 0.18 \mathrm{~mm}$ 


\section{Data collection}

Bruker APEXII CCD

diffractometer

Radiation source: fine-focus sealed tube

Graphite monochromator

$\varphi$ and $\omega$ scans

Absorption correction: multi-scan

(SADABS; Bruker, 2009)

$T_{\min }=0.965, T_{\max }=0.986$

\section{Refinement}

Refinement on $F^{2}$

Least-squares matrix: full

$R\left[F^{2}>2 \sigma\left(F^{2}\right)\right]=0.056$

$w R\left(F^{2}\right)=0.166$

$S=1.14$

2864 reflections

155 parameters

0 restraints

Primary atom site location: structure-invariant direct methods
18116 measured reflections

2864 independent reflections

2463 reflections with $I>2 \sigma(I)$

$R_{\text {int }}=0.056$

$\theta_{\max }=28.3^{\circ}, \theta_{\min }=1.6^{\circ}$

$h=-17 \rightarrow 17$

$k=-7 \rightarrow 7$

$l=-21 \rightarrow 22$

Secondary atom site location: difference Fourier map

Hydrogen site location: inferred from

neighbouring sites

$\mathrm{H}$-atom parameters constrained

$w=1 /\left[\sigma^{2}\left(F_{\mathrm{o}}^{2}\right)+(0.0577 P)^{2}+1.3715 P\right]$

where $P=\left(F_{\mathrm{o}}{ }^{2}+2 F_{\mathrm{c}}{ }^{2}\right) / 3$

$(\Delta / \sigma)_{\max }<0.001$

$\Delta \rho_{\max }=0.29 \mathrm{e} \AA^{-3}$

$\Delta \rho_{\min }=-0.31$ e $\AA^{-3}$

Special details

Geometry. All e.s.d.'s (except the e.s.d. in the dihedral angle between two l.s. planes) are estimated using the full covariance matrix. The cell e.s.d.'s are taken into account individually in the estimation of e.s.d.'s in distances, angles and torsion angles; correlations between e.s.d.'s in cell parameters are only used when they are defined by crystal symmetry. An approximate (isotropic) treatment of cell e.s.d.'s is used for estimating e.s.d.'s involving 1.s. planes.

Refinement. Refinement of $F^{2}$ against ALL reflections. The weighted $R$-factor $w R$ and goodness of fit $S$ are based on $F^{2}$, conventional $R$-factors $R$ are based on $F$, with $F$ set to zero for negative $F^{2}$. The threshold expression of $F^{2}>\sigma\left(F^{2}\right)$ is used only for calculating $R$-factors(gt) $e t c$. and is not relevant to the choice of reflections for refinement. $R$-factors based on $F^{2}$ are statistically about twice as large as those based on $F$, and $R$ - factors based on ALL data will be even larger.

Fractional atomic coordinates and isotropic or equivalent isotropic displacement parameters $\left(\AA^{2}\right)$

\begin{tabular}{lllll}
\hline & $x$ & $y$ & $z$ & $U_{\text {iso }} * / U_{\text {eq }}$ \\
\hline $\mathrm{N} 1$ & $0.64381(12)$ & $0.1086(3)$ & $0.81833(9)$ & $0.0210(3)$ \\
$\mathrm{H} 1 \mathrm{~N}$ & 0.5882 & 0.0697 & 0.7834 & $0.025^{*}$ \\
$\mathrm{~N} 2$ & $0.70730(12)$ & $0.3584(3)$ & $0.93020(9)$ & $0.0201(3)$ \\
$\mathrm{N} 3$ & $0.53921(12)$ & $0.4215(3)$ & $0.84955(9)$ & $0.0206(3)$ \\
$\mathrm{C} 1$ & $0.83033(14)$ & $0.0059(4)$ & $0.85440(11)$ & $0.0246(4)$ \\
$\mathrm{H} 1$ & 0.8436 & 0.1437 & 0.8906 & $0.030^{*}$ \\
$\mathrm{C} 2$ & $0.90995(15)$ & $-0.1513(4)$ & $0.84207(12)$ & $0.0277(4)$ \\
$\mathrm{H} 2$ & 0.9778 & -0.1183 & 0.8700 & $0.033^{*}$ \\
$\mathrm{C} 3$ & $0.89324(15)$ & $-0.3555(4)$ & $0.79011(12)$ & $0.0256(4)$ \\
$\mathrm{H} 3$ & 0.9486 & -0.4621 & 0.7827 & $0.031^{*}$ \\
$\mathrm{C} 4$ & $0.79368(15)$ & $-0.4010(4)$ & $0.74907(11)$ & $0.0241(4)$ \\
$\mathrm{H} 4$ & 0.7808 & -0.5396 & 0.7131 & $0.029^{*}$ \\
$\mathrm{C} 5$ & $0.71331(14)$ & $-0.2457(4)$ & $0.76022(11)$ & $0.0208(4)$ \\
$\mathrm{H} 5$ & 0.6458 & -0.2787 & 0.7318 & $0.025^{*}$ \\
C6 & $0.73029(14)$ & $-0.0402(4)$ & $0.81301(10)$ & $0.0195(4)$ \\
C7 & $0.63140(14)$ & $0.3035(4)$ & $0.86853(10)$ & $0.0189(4)$
\end{tabular}




$\begin{array}{lllll}\text { C8 } & 0.69016(14) & 0.5539(4) & 0.97648(10) & 0.0208(4) \\ \text { C9 } & 0.59859(15) & 0.6871(4) & 0.96202(11) & 0.0233(4) \\ \text { H9 } & 0.5873 & 0.8251 & 0.9951 & 0.028^{*} \\ \text { C10 } & 0.52353(14) & 0.6127(4) & 0.89742(11) & 0.0209(4) \\ \text { C11 } & 0.77222(15) & 0.6184(4) & 1.04550(11) & 0.0246(4) \\ \text { H11 } & 0.7567 & 0.7622 & 1.0796 & 0.029^{*} \\ \text { C12 } & 0.83749(15) & 0.4101(4) & 1.08962(12) & 0.0276(4) \\ \text { H12A } & 0.8585 & 0.4258 & 1.1488 & 0.033^{*} \\ \text { H12B } & 0.8239 & 0.2360 & 1.0698 & 0.033^{*} \\ \text { C13 } & 0.88371(16) & 0.5880(4) & 1.03804(12) & 0.0300(5) \\ \text { H13A } & 0.8989 & 0.5239 & 0.9863 & 0.036^{*} \\ \text { H13B } & 0.9334 & 0.7136 & 1.0653 & 0.036^{*} \\ \text { C14 } & 0.42255(15) & 0.7483(4) & 0.87894(12) & 0.0255(4) \\ \text { H14A } & 0.4283 & 0.8895 & 0.8427 & 0.038^{*} \\ \text { H14B } & 0.4043 & 0.8118 & 0.9291 & 0.038^{*} \\ \text { H14C } & 0.3690 & 0.6324 & 0.8530 & 0.038^{*}\end{array}$

Atomic displacement parameters $\left(\AA^{2}\right)$

\begin{tabular}{lllllll}
\hline & $U^{11}$ & $U^{22}$ & $U^{33}$ & $U^{12}$ & $U^{13}$ & $U^{23}$ \\
\hline $\mathrm{N} 1$ & $0.0185(7)$ & $0.0259(8)$ & $0.0165(7)$ & $0.0004(6)$ & $-0.0023(5)$ & $-0.0027(6)$ \\
$\mathrm{N} 2$ & $0.0205(7)$ & $0.0238(8)$ & $0.0145(7)$ & $0.0001(6)$ & $-0.0009(5)$ & $0.0008(6)$ \\
$\mathrm{N} 3$ & $0.0196(7)$ & $0.0250(8)$ & $0.0164(7)$ & $0.0014(6)$ & $0.0010(5)$ & $0.0005(6)$ \\
$\mathrm{C} 1$ & $0.0218(9)$ & $0.0278(10)$ & $0.0221(9)$ & $-0.0006(8)$ & $-0.0016(7)$ & $-0.0040(8)$ \\
$\mathrm{C} 2$ & $0.0209(9)$ & $0.0329(11)$ & $0.0270(9)$ & $0.0014(8)$ & $-0.0016(7)$ & $-0.0027(8)$ \\
$\mathrm{C} 3$ & $0.0242(9)$ & $0.0279(10)$ & $0.0243(9)$ & $0.0047(8)$ & $0.0031(7)$ & $0.0001(8)$ \\
$\mathrm{C} 4$ & $0.0290(10)$ & $0.0234(10)$ & $0.0194(8)$ & $-0.0003(8)$ & $0.0027(7)$ & $-0.0025(7)$ \\
$\mathrm{C} 5$ & $0.0226(9)$ & $0.0220(9)$ & $0.0166(8)$ & $-0.0016(7)$ & $0.0002(6)$ & $0.0006(7)$ \\
C6 & $0.0207(8)$ & $0.0227(9)$ & $0.0146(7)$ & $0.0004(7)$ & $0.0014(6)$ & $0.0019(7)$ \\
C7 & $0.0200(8)$ & $0.0221(9)$ & $0.0138(7)$ & $0.0002(7)$ & $0.0007(6)$ & $0.0016(7)$ \\
C8 & $0.0227(9)$ & $0.0233(9)$ & $0.0151(8)$ & $-0.0009(7)$ & $0.0005(6)$ & $0.0016(7)$ \\
C9 & $0.0258(9)$ & $0.0246(10)$ & $0.0181(8)$ & $0.0028(8)$ & $0.0001(7)$ & $-0.0033(7)$ \\
C10 & $0.0219(9)$ & $0.0235(9)$ & $0.0167(8)$ & $0.0015(7)$ & $0.0018(6)$ & $0.0022(7)$ \\
C11 & $0.0250(9)$ & $0.0284(10)$ & $0.0180(8)$ & $0.0005(8)$ & $-0.0025(7)$ & $-0.0035(7)$ \\
C12 & $0.0244(9)$ & $0.0333(11)$ & $0.0216(9)$ & $0.0006(8)$ & $-0.0053(7)$ & $0.0005(8)$ \\
C13 & $0.0245(9)$ & $0.0382(12)$ & $0.0247(9)$ & $-0.0057(8)$ & $-0.0022(7)$ & $-0.0022(9)$ \\
C14 & $0.0232(9)$ & $0.0289(10)$ & $0.0226(9)$ & $0.0054(8)$ & $-0.0005(7)$ & $-0.0004(8)$ \\
& & & & & &
\end{tabular}

Geometric parameters $\left(\AA,{ }^{\circ}\right)$

\begin{tabular}{llll}
\hline $\mathrm{N} 1-\mathrm{C} 7$ & $1.367(2)$ & $\mathrm{C} 5-\mathrm{H} 5$ & 0.9500 \\
$\mathrm{~N} 1-\mathrm{C} 6$ & $1.404(2)$ & $\mathrm{C} 8-\mathrm{C} 9$ & $1.384(3)$ \\
$\mathrm{N} 1-\mathrm{H} 1 \mathrm{~N}$ & 0.8800 & $\mathrm{C} 8-\mathrm{C} 11$ & $1.481(2)$ \\
$\mathrm{N} 2-\mathrm{C} 7$ & $1.341(2)$ & $\mathrm{C} 9-\mathrm{C} 10$ & $1.392(3)$ \\
$\mathrm{N} 2-\mathrm{C} 8$ & $1.343(2)$ & $\mathrm{C} 9-\mathrm{H} 9$ & 0.9500 \\
$\mathrm{~N} 3-\mathrm{C} 10$ & $1.337(2)$ & $\mathrm{C} 10-\mathrm{C} 14$ & $1.498(3)$ \\
$\mathrm{N} 3-\mathrm{C} 7$ & $1.355(2)$ & $\mathrm{C} 11-\mathrm{C} 13$ & $1.507(3)$ \\
$\mathrm{C} 1-\mathrm{C} 2$ & $1.387(3)$ & $\mathrm{C} 11-\mathrm{C} 12$ & $1.514(3)$
\end{tabular}




\begin{tabular}{|c|c|c|c|}
\hline $\mathrm{C} 1-\mathrm{C} 6$ & $1.400(2)$ & $\mathrm{C} 11-\mathrm{H} 11$ & 1.0000 \\
\hline $\mathrm{C} 1-\mathrm{H} 1$ & 0.9500 & $\mathrm{C} 12-\mathrm{C} 13$ & $1.488(3)$ \\
\hline $\mathrm{C} 2-\mathrm{C} 3$ & $1.388(3)$ & $\mathrm{C} 12-\mathrm{H} 12 \mathrm{~A}$ & 0.9900 \\
\hline $\mathrm{C} 2-\mathrm{H} 2$ & 0.9500 & $\mathrm{C} 12-\mathrm{H} 12 \mathrm{~B}$ & 0.9900 \\
\hline $\mathrm{C} 3-\mathrm{C} 4$ & $1.392(3)$ & $\mathrm{C} 13-\mathrm{H} 13 \mathrm{~A}$ & 0.9900 \\
\hline $\mathrm{C} 3-\mathrm{H} 3$ & 0.9500 & $\mathrm{C} 13-\mathrm{H} 13 \mathrm{~B}$ & 0.9900 \\
\hline $\mathrm{C} 4-\mathrm{C} 5$ & $1.383(3)$ & $\mathrm{C} 14-\mathrm{H} 14 \mathrm{~A}$ & 0.9800 \\
\hline $\mathrm{C} 4-\mathrm{H} 4$ & 0.9500 & C14-H14B & 0.9800 \\
\hline $\mathrm{C} 5-\mathrm{C} 6$ & $1.402(3)$ & $\mathrm{C} 14-\mathrm{H} 14 \mathrm{C}$ & 0.9800 \\
\hline $\mathrm{C} 7-\mathrm{N} 1-\mathrm{C} 6$ & $130.97(15)$ & $\mathrm{C} 8-\mathrm{C} 9-\mathrm{H} 9$ & 121.0 \\
\hline $\mathrm{C} 7-\mathrm{N} 1-\mathrm{H} 1 \mathrm{~N}$ & 114.5 & $\mathrm{C} 10-\mathrm{C} 9-\mathrm{H} 9$ & 121.0 \\
\hline $\mathrm{C} 6-\mathrm{N} 1-\mathrm{H} 1 \mathrm{~N}$ & 114.5 & $\mathrm{~N} 3-\mathrm{C} 10-\mathrm{C} 9$ & $121.56(17)$ \\
\hline $\mathrm{C} 7-\mathrm{N} 2-\mathrm{C} 8$ & $116.06(16)$ & $\mathrm{N} 3-\mathrm{C} 10-\mathrm{C} 14$ & $117.93(16)$ \\
\hline $\mathrm{C} 10-\mathrm{N} 3-\mathrm{C} 7$ & $116.01(16)$ & $\mathrm{C} 9-\mathrm{C} 10-\mathrm{C} 14$ & $120.50(17)$ \\
\hline $\mathrm{C} 2-\mathrm{C} 1-\mathrm{C} 6$ & $119.45(18)$ & $\mathrm{C} 8-\mathrm{C} 11-\mathrm{C} 13$ & $119.78(16)$ \\
\hline $\mathrm{C} 2-\mathrm{C} 1-\mathrm{H} 1$ & 120.3 & $\mathrm{C} 8-\mathrm{C} 11-\mathrm{C} 12$ & $119.22(18)$ \\
\hline $\mathrm{C} 6-\mathrm{C} 1-\mathrm{H} 1$ & 120.3 & $\mathrm{C} 13-\mathrm{C} 11-\mathrm{C} 12$ & $58.99(14)$ \\
\hline $\mathrm{C} 1-\mathrm{C} 2-\mathrm{C} 3$ & $121.81(18)$ & $\mathrm{C} 8-\mathrm{C} 11-\mathrm{H} 11$ & 115.7 \\
\hline $\mathrm{C} 1-\mathrm{C} 2-\mathrm{H} 2$ & 119.1 & $\mathrm{C} 13-\mathrm{C} 11-\mathrm{H} 11$ & 115.7 \\
\hline $\mathrm{C} 3-\mathrm{C} 2-\mathrm{H} 2$ & 119.1 & $\mathrm{C} 12-\mathrm{C} 11-\mathrm{H} 11$ & 115.7 \\
\hline $\mathrm{C} 2-\mathrm{C} 3-\mathrm{C} 4$ & $118.62(18)$ & $\mathrm{C} 13-\mathrm{C} 12-\mathrm{C} 11$ & $60.26(14)$ \\
\hline $\mathrm{C} 2-\mathrm{C} 3-\mathrm{H} 3$ & 120.7 & $\mathrm{C} 13-\mathrm{C} 12-\mathrm{H} 12 \mathrm{~A}$ & 117.7 \\
\hline $\mathrm{C} 4-\mathrm{C} 3-\mathrm{H} 3$ & 120.7 & $\mathrm{C} 11-\mathrm{C} 12-\mathrm{H} 12 \mathrm{~A}$ & 117.7 \\
\hline $\mathrm{C} 5-\mathrm{C} 4-\mathrm{C} 3$ & $120.47(18)$ & $\mathrm{C} 13-\mathrm{C} 12-\mathrm{H} 12 \mathrm{~B}$ & 117.7 \\
\hline $\mathrm{C} 5-\mathrm{C} 4-\mathrm{H} 4$ & 119.8 & $\mathrm{C} 11-\mathrm{C} 12-\mathrm{H} 12 \mathrm{~B}$ & 117.7 \\
\hline $\mathrm{C} 3-\mathrm{C} 4-\mathrm{H} 4$ & 119.8 & $\mathrm{H} 12 \mathrm{~A}-\mathrm{C} 12-\mathrm{H} 12 \mathrm{~B}$ & 114.9 \\
\hline $\mathrm{C} 4-\mathrm{C} 5-\mathrm{C} 6$ & $120.82(17)$ & $\mathrm{C} 12-\mathrm{C} 13-\mathrm{C} 11$ & $60.74(14)$ \\
\hline $\mathrm{C} 4-\mathrm{C} 5-\mathrm{H} 5$ & 119.6 & $\mathrm{C} 12-\mathrm{C} 13-\mathrm{H} 13 \mathrm{~A}$ & 117.7 \\
\hline $\mathrm{C} 6-\mathrm{C} 5-\mathrm{H} 5$ & 119.6 & $\mathrm{C} 11-\mathrm{C} 13-\mathrm{H} 13 \mathrm{~A}$ & 117.7 \\
\hline $\mathrm{C} 1-\mathrm{C} 6-\mathrm{C} 5$ & $118.84(17)$ & $\mathrm{C} 12-\mathrm{C} 13-\mathrm{H} 13 \mathrm{~B}$ & 117.7 \\
\hline $\mathrm{C} 1-\mathrm{C} 6-\mathrm{N} 1$ & $125.02(17)$ & $\mathrm{C} 11-\mathrm{C} 13-\mathrm{H} 13 \mathrm{~B}$ & 117.7 \\
\hline $\mathrm{C} 5-\mathrm{C} 6-\mathrm{N} 1$ & $116.12(16)$ & $\mathrm{H} 13 \mathrm{~A}-\mathrm{C} 13-\mathrm{H} 13 \mathrm{~B}$ & 114.8 \\
\hline $\mathrm{N} 2-\mathrm{C} 7-\mathrm{N} 3$ & $126.65(17)$ & $\mathrm{C} 10-\mathrm{C} 14-\mathrm{H} 14 \mathrm{~A}$ & 109.5 \\
\hline $\mathrm{N} 2-\mathrm{C} 7-\mathrm{N} 1$ & $119.35(16)$ & $\mathrm{C} 10-\mathrm{C} 14-\mathrm{H} 14 \mathrm{~B}$ & 109.5 \\
\hline $\mathrm{N} 3-\mathrm{C} 7-\mathrm{N} 1$ & $114.00(16)$ & $\mathrm{H} 14 \mathrm{~A}-\mathrm{C} 14-\mathrm{H} 14 \mathrm{~B}$ & 109.5 \\
\hline $\mathrm{N} 2-\mathrm{C} 8-\mathrm{C} 9$ & $121.73(17)$ & $\mathrm{C} 10-\mathrm{C} 14-\mathrm{H} 14 \mathrm{C}$ & 109.5 \\
\hline $\mathrm{N} 2-\mathrm{C} 8-\mathrm{C} 11$ & $117.43(17)$ & $\mathrm{H} 14 \mathrm{~A}-\mathrm{C} 14-\mathrm{H} 14 \mathrm{C}$ & 109.5 \\
\hline $\mathrm{C} 9-\mathrm{C} 8-\mathrm{C} 11$ & $120.82(17)$ & $\mathrm{H} 14 \mathrm{~B}-\mathrm{C} 14-\mathrm{H} 14 \mathrm{C}$ & 109.5 \\
\hline $\mathrm{C} 8-\mathrm{C} 9-\mathrm{C} 10$ & $117.96(18)$ & & \\
\hline $\mathrm{C} 6-\mathrm{C} 1-\mathrm{C} 2-\mathrm{C} 3$ & $-0.5(3)$ & $\mathrm{C} 6-\mathrm{N} 1-\mathrm{C} 7-\mathrm{N} 3$ & $172.68(17)$ \\
\hline $\mathrm{C} 1-\mathrm{C} 2-\mathrm{C} 3-\mathrm{C} 4$ & $0.5(3)$ & $\mathrm{C} 7-\mathrm{N} 2-\mathrm{C} 8-\mathrm{C} 9$ & $1.2(3)$ \\
\hline $\mathrm{C} 2-\mathrm{C} 3-\mathrm{C} 4-\mathrm{C} 5$ & $-0.2(3)$ & $\mathrm{C} 7-\mathrm{N} 2-\mathrm{C} 8-\mathrm{C} 11$ & $179.58(16)$ \\
\hline $\mathrm{C} 3-\mathrm{C} 4-\mathrm{C} 5-\mathrm{C} 6$ & $-0.1(3)$ & $\mathrm{N} 2-\mathrm{C} 8-\mathrm{C} 9-\mathrm{C} 10$ & $0.0(3)$ \\
\hline $\mathrm{C} 2-\mathrm{C} 1-\mathrm{C} 6-\mathrm{C} 5$ & $0.2(3)$ & $\mathrm{C} 11-\mathrm{C} 8-\mathrm{C} 9-\mathrm{C} 10$ & $-178.32(18)$ \\
\hline $\mathrm{C} 2-\mathrm{C} 1-\mathrm{C} 6-\mathrm{N} 1$ & $-178.05(18)$ & $\mathrm{C} 7-\mathrm{N} 3-\mathrm{C} 10-\mathrm{C} 9$ & $1.0(3)$ \\
\hline $\mathrm{C} 4-\mathrm{C} 5-\mathrm{C} 6-\mathrm{C} 1$ & $0.1(3)$ & $\mathrm{C} 7-\mathrm{N} 3-\mathrm{C} 10-\mathrm{C} 14$ & $-179.81(17)$ \\
\hline
\end{tabular}


supporting information

$\begin{array}{llll}\mathrm{C} 4-\mathrm{C} 5-\mathrm{C} 6-\mathrm{N} 1 & 178.52(17) & \mathrm{C} 8-\mathrm{C} 9-\mathrm{C} 10-\mathrm{N} 3 & -1.2(3) \\ \mathrm{C} 7-\mathrm{N} 1-\mathrm{C} 6-\mathrm{C} 1 & -8.8(3) & \mathrm{C} 8-\mathrm{C} 9-\mathrm{C} 10-\mathrm{C} 14 & 179.64(18) \\ \mathrm{C} 7-\mathrm{N} 1-\mathrm{C} 6-\mathrm{C} 5 & 172.85(18) & \mathrm{N} 2-\mathrm{C} 8-\mathrm{C} 11-\mathrm{C} 13 & 35.3(3) \\ \mathrm{C} 8-\mathrm{N} 2-\mathrm{C} 7-\mathrm{N} 3 & -1.5(3) & \mathrm{C} 9-\mathrm{C} 8-\mathrm{C} 11-\mathrm{C} 13 & -146.3(2) \\ \mathrm{C} 8-\mathrm{N} 2-\mathrm{C} 7-\mathrm{N} 1 & 179.34(16) & \mathrm{N} 2-\mathrm{C} 8-\mathrm{C} 11-\mathrm{C} 12 & -33.6(3) \\ \mathrm{C} 10-\mathrm{N} 3-\mathrm{C} 7-\mathrm{N} 2 & 0.4(3) & \mathrm{C} 9-\mathrm{C} 8-\mathrm{C} 11-\mathrm{C} 12 & 144.84(19) \\ \mathrm{C} 10-\mathrm{N} 3-\mathrm{C} 7-\mathrm{N} 1 & 179.63(16) & \mathrm{C} 8-\mathrm{C} 11-\mathrm{C} 12-\mathrm{C} 13 & 109.1(2) \\ \mathrm{C} 6-\mathrm{N} 1-\mathrm{C} 7-\mathrm{N} 2 & -8.0(3) & \mathrm{C} 8-\mathrm{C} 11-\mathrm{C} 13-\mathrm{C} 12 & -108.2(2)\end{array}$

